\title{
Analysis on the MRI and BAEP Results of Neonatal Brain with Different Levels of Bilirubin
}

\section{LUZHONGXING ( $\nabla$ szdxIzx@126.com )}

Changzhou Maternal And Child Health Care Hospital https://orcid.org/0000-0002-4383-1475

\section{SHOULING DING}

Paediatric Department of Taicang First People's Hospital

\section{FEN WANG}

Paediatic Department of Taicang First People's Hospital

Haitao Lv

Children's Hospital of Soochow University

\section{Research article}

Keywords: MRI abnormality change, acute bilirubin encephalopathy, bilirubin, hyperbilirubinemia, BAEP

Posted Date: June 25th, 2020

DOl: https://doi.org/10.21203/rs.3.rs-17458/v2

License: (c) (i) This work is licensed under a Creative Commons Attribution 4.0 International License. Read Full License 


\section{Abstract}

BackgroundXTo explore whether there is abnormality of neonatal brains' MRI and BAEP with different bilirubin levels, and to provide an objective basis for early diagnosis on the bilirubin induced subclinical damage on brains.

Methods: To retrospectively analyze the clinical data of 103 neonatal patients, who had been hospitalized in Neonatology Department of Taicang First People's Hospital from March 2013 to September 2015, to conduct routine brain MRI examination, BAEP testing and to analyze BAEP and MRI image results of the neonatal patients, who were divided into three groups based on the levels of total serum bilirubin concentration (TSB), 16 cases in mild group (TSB:0.0 229.0 $\mu \mathrm{mol} / \mathrm{L}$ ), 49 cases in moderate group (TSB: 229.0 342.0 $\mu \mathrm{mol} / \mathrm{L})$ and 38 cases in severe group (TSB $\geq 342.0 \mu \mathrm{mol} / \mathrm{L})$;

Results: We found as follows: A. Comparison of the bilirubin value of the different group : 1 . The bilirubin value of the mild group is $171.99 \pm 33.50 \mu \mathrm{mol} / \mathrm{L}$, the moderate group is $293.98 \pm 32.09 \mu \mathrm{mol} / \mathrm{L}$, and the severe group is $375.59 \pm 34.25 \mu \mathrm{mol} / \mathrm{L}$. And the comparison of bilirubin values of the three groups of neonates (Pख0.01) indicates the difference is statistically significant (P囚0.01). 2. The bilirubin value of the pre-term group is $289.70 \pm 85.38 \mu \mathrm{mol} / \mathrm{Land}$ the full-term group is $310.36 \pm 72.32 \mu \mathrm{mol} / \mathrm{L}$, but the comparison of the bilirubin values between pre-term group and full-term group indicates that the difference is not statistically significant (Pख0.05).3. The bilirubin value of the normal brain MRI group(82) is $305.55 \pm 74.54 \mu \mathrm{mol} / \mathrm{L}$ and the abnormal brain MRI group is $303.56 \pm 83.04 \mu \mathrm{mol} / \mathrm{L}$; the comparison of bilirubin values between the two groups indicates that the difference is not statistically significant $(\mathrm{P} \bigotimes$ 0.05 ). B. The weight value of the $\varangle 2500 \mathrm{~g}$ group is $2.04 \pm 0.21$ and the $\geq 2500 \mathrm{ggroup}$ is $3.39 \pm 0.46$; the weight comparison of the two groups indicates that the difference is statistically significant (Pख0.01). C. Comparison of the abnormal MRI of the different groups: 1.The brain MRI result's abnormal ratio of the mild group is $31.25 \%$, the moderate group is $16.33 \%$ and the severe group is $21.05 \%$, but the comparison of brain MRI results of the three neonates groups indicates that the difference is not statistically significant (P凶0.05). 2. The brain MRI result's abnormal ratio of the pre-term is $30.77 \%$ and the full-term group is $16.88 \%$, but the comparison of brain MRI results between prem-term group and full-term group indicates that the difference is not statistically significant (Pख0.05). 3.The brain MRI result's abnormal ratio of the $₫ 2500 \mathrm{~g}$ group is $37.50 \%$ and the $\geq 2500 \mathrm{~g}$ group is $17.24 \%$; but the comparison of brain MRI results of two neonates groups indicates that the difference is not statistically significant(Pख0.05). D. Comparison of abnormal MRI signal values of globus pallidus on T1WI in different groups: 1 . The comparison of normal group signal values with that of mild group $(p<0.05)$, with that of moderate group and with that of severe group $(p<0.01)$ indicates that the difference is statistically significant; 2 . The comparison of signal values between mild and moderate groups $(p<0.05)$ and between mild group and severe group $(p<0.01)$ indicates that the difference is statistically significant; 3 . The comparison of signal values between moderate group and severe group indicates that the difference is statistically significant $(p<0.05)$. E. Comparison of BAEP testing results in groups: 1 . There were $27(26.21 \%)$ cases in abnormalities of the BAEP results of all 103cases bilirubin patients. 2. There were $15(18.29 \%)$ cases in abnormalities of the BAEP result of the 82 cases normal brain MRL , 2(40\%) cases in abnormalities of the 
BAEP result of the 5 cases abnormal MRI in mild bilirubin group, $4 \llbracket 50 \% \rrbracket$ cases in abnormalities of the

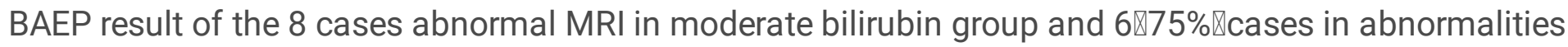
of the BAEP result of the 8 cases abnormal brain MRI in severe bilirubin group. 3. After one month review of the BAEP result, there was $0 \otimes 0.00 \% \bigotimes$ abnormal case in the normal MRI and the mild group; there were 1 $\triangle 20 \% \bigotimes$ abnormal case in the moderate group and $2 \varangle 25 \% \bigotimes$ cases in the severe group.

Conclusion: At low level of bilirubin, central nervous system damage may also occur and can be detected as abnormality by MRI and BAEP. Meanwhile, MRI and BAEP can also provide early abnormal information for the judgment of central nervous system damage of the children with NHB who have no acute bilirubin encephalopathy (ABE) clinical features, and provide clues for early treatment and early intervention.

\section{Background}

Although neonatal jaundice is quite common, affecting $60 \%-80 \%$ of newborns overall ${ }^{[1]}$. Mainly, with the high accumulation of unconjugated bilirubin, severe patients (their bilirubin's concentration $>20 \mathrm{mg} / \mathrm{dL}$ ) may suffer from bilirubinencephalopathy or kernicterus, and bilirubun-induced neurological dysfunction $(B I N D)^{[2]}$, which will cause permanent damage to the basal ganglia, hippocampus, hypothalamus, cerebellar neurons, brainstem ${ }^{[3,4]}$, etc. Clinical manifestation includes lethargy, poor response, fever, apnea, convulsion, auditory complications and so on ${ }^{[2]}$. Magnetic resonance imaging (MRI) and hearing screening have proved to be highly sensitive in bilirubin encephalopathy. With MRI, high intensity signals of globus pallidus and subthalamic nucleus can be detected in early T1WI, high stripy symmetric signals of globus pallidus can be detected in later $\mathrm{T} 2 \mathrm{~W} \mathrm{I}^{[5,6]}$. And abnormal changes occur in the BAER ${ }^{[7]}$. However, severe hyperbilirubinemia ( $>20 \mathrm{mg} / \mathrm{dL}$ ) that could potentially lead to bilirubin encephalopathy or kernicterus and neurodevelopmental complications is said to be much rarer, affecting less than $2 \%$ of newborn infants ${ }^{[8]}$, most of them have no clinical manifestation except jaundice. It is still debated whether these lower levels of hyperbilirubinemia cause brain damage to neonates or not? It is also unclear whether different degrees of hyperbilirubinemia result in different patterns of brain injury? For answering these questions, we examined the brain MRI and hearing screening of neonates with different levels of bilirubin levels.

\section{Methods}

\subsection{Methods and Criteria}

\subsubsection{Methods}

MRI: A Siemens Skyra 3.0T superconductivity magnetic resonance scanner and new Tim4G platform equipped with 20 units of high-density matrix head and neck joint coils was used for this study. Before MRI scanning, the neonate patients were given routine sedation, kept in supine position and warm. 
$\mathrm{T} 1 \mathrm{WI}(\mathrm{TR}=477 \mathrm{~ms}, \mathrm{TE}=15 \mathrm{~ms}), \mathrm{T} 2 \mathrm{WI}(\mathrm{TR}=4060 \mathrm{~ms}, \mathrm{TE}=120 \mathrm{~ms})$, matrix 256×256, slice thickness $5 \mathrm{~mm}$, gap $0.4 \mathrm{~mm}$. Images were obtained, coronal scanning was done for all neonate patients.

\subsubsection{Criteria for Diagnosis}

All MRI results were jointly written and analyzed by two radiologists who did not know the clinical history of patient.

MRI: high intensity signals of globus pallidus and subthalamic nucleus detected in $\mathrm{T} 1 \mathrm{WI}$, or high intensity symmetric signals of globus pallidus detected in T2WI or uneven signal of basal ganglion was considered abnormal. No above-mentioned manifestation was regarded as normality ${ }^{[5,6]}$.

\subsubsection{Brainstem Auditory Evoked Potential (BAEP)}

BAEP testing was performed by an otolaryngologist skilled in BAEP technology in a quiet environment.

\subsubsection{Criteria for Diagnosis}

BAEP: The results were considered to be abnormal as shown that the incubation period of I wave, III wave and $V$ wave is prolonged, the interphase period of $\mathrm{I} \sim \mathrm{V}$ wave is prolonged, the waveform is poorly differentiated or disappears, and the single or bilateral I wave, III wave and $V$ wave all disappear ${ }^{[7]}$.

\subsection{Statistical Method}

Data analysis was performed by the use of SPSS 23.0 statistical software. Each measuring parameter was expressed by Mean $\pm S D$ and t-tests (multi-group comparisons), while each counting parameter was expressed by rate and $\square^{2}$ tests(comparisons among groups). $P<0.05$, the difference is statistically significant.

\section{Results}

\subsection{Clinical Data}

We retrospectively analyzed 103 neonates(51 boys and 52 girls) that we assume these neonates have no "clinical" manifestation of bilirubin encephalopathy, who had been hospitalized in Neonatology Department of Taicang First People's Hospital from March 2013 to September 2015. Their gestational ages ranged from $31+6$ weeks to $43+1$ weeks(in average,38.14 \pm 2.21 weeks), birth weight from $1.55 \mathrm{~kg}$ to $5.00 \mathrm{~kg}$ (average,3.18 $\pm 0.64 \mathrm{~kg}), 26$ pre-term neonates and77 full-term neonates. These neonates were divided into three groups based on their levels of serum bilirubin concentration, 16 cases in mild group(TSB: $0.0 \sim 229.0 \mu \mathrm{mol} / \mathrm{L}$ ), 49 cases in moderate group (TSB: 229.0 $342.0 \mu \mathrm{mol} / \mathrm{L}$ ) and 38 cases in severe group ( $T S B \geq 342.0 \mu \mathrm{mol} / \mathrm{L})$. Possible diseases which have manifestations similar to that of bilirubin encephalopathy, such as toxic cerebral hypoxia damage, hepatolenticular degeneration, hypoxic ischemic encephalopathy, intracranial hemorrhage, hypoglycemia, etc. were excluded. 
2.2.1 Comparison of the bilirubin value of the different group

Table 1

\begin{tabular}{lccc} 
Group & Bilirubin value & $t$ & $\mathrm{p}$ \\
\hline Mild group & \multicolumn{1}{c}{$171.99 \pm 33.50$} & 20.54 & 0.00 \\
\hline Moderate group & $293.98 \pm 32.09$ & 64.13 & 0.00 \\
\hline Severe group & $375.59 \pm 34.25$ & 67.59 & 0.00 \\
\hline & & & \\
\hline Pre-term & & & \\
\hline Full-term & $289.70 \pm 85.38$ & -1.20 & 0.232 \\
\hline & $310.36 \pm 72.32$ & & \\
\hline Normal MRI & & & \\
\hline Abnormal MRI $^{3}$ & $305.55 \pm 74.54$ & 0.11 & 0.92 \\
\hline
\end{tabular}

The bilirubin value of the mild group is $171.99 \pm 33.50 \mu \mathrm{mol} / \mathrm{L}$, the moderate group is $293.98 \pm 32.09$ $\mu \mathrm{mol} / \mathrm{L}$,and the severe group is $375.59 \pm 34.25 \mu \mathrm{mol} / \mathrm{L}$. And the comparison of bilirubin values of the three groups of neonates $(\mathrm{P} \otimes 0.01)$ indicates the difference is statistically significant. 2 . The bilirubin value of the pre-term group is $289.70 \pm 85.38 \mu \mathrm{mol} / \mathrm{L}$ and the full-term group is $310.36 \pm 72.32 \mu \mathrm{mol} / \mathrm{L}$, but the comparison of the bilirubin values between pre-term group and full-term group ( $P \otimes 0.05)$ indicates that the difference is not statistically significant.3. The bilirubin value of the normal brain MRI group(82) is $305.55 \pm 74.54 \mu \mathrm{mol} / \mathrm{L}$ and the abnormal brain MRI group is $303.56 \pm 83.04 \mu \mathrm{mol} / \mathrm{L}$; thecomparison of bilirubin values between the two groups $(\mathrm{P} \otimes 0.05)$ indicates that the difference is not statistically significant.

2.2.2 Two groups divided based on the weight of $2500 \mathrm{~g}$

Table 2

\begin{tabular}{llll} 
Group & $\mathbf{0 2 5 0 0 g}$ & & $\mathbf{2 5 0 0 \mathrm { g }}$ \\
\hline Weight & $2.04 \pm 0.21$ & & $3.39 \pm 0.46$ \\
\hline $\mathrm{t}$ & & -18.85 & \\
\hline $\mathrm{P}$ & & 0.00 &
\end{tabular}


The weight value of the $\nabla 2500 \mathrm{~g}$ group is $2.04 \pm 0.21$ and the $\geq 2500 \mathrm{ggroup}$ is $3.39 \pm 0.46$; the weight comparison of the two groups indicates that the difference is statistically significant(Pख0.01).

2.2.3 Comparison of the abnormal MRI of the different groups

Table 3

\begin{tabular}{lllll} 
Group & Normal MRI & Abnormal MRI & $\mathbb{\bigotimes}^{2}$ & $p$ \\
\hline Mild group & $11(68.75 \%)$ & $5(31.25 \%)$ & & \\
\hline Moderate group & $41(83.67 \%)$ & $8(16.33 \%)$ & 1.579 & 0.454 \\
\hline Severe group & $30(78.95 \%)$ & $8(21.05 \%)$ & & \\
\hline & & & & \\
\hline Pre-term ${ }^{2}$ & $18(69.23 \%)$ & $8(30.77 \%)$ & 2.310 & 0.160 \\
\hline Full-term & $64(83.12 \%)$ & $13(16.88 \%)$ & & \\
\hline & & & & \\
\hline$<2500 g^{3}$ & $10(62.50 \%)$ & $6(37.50 \%)$ & 3.420 & 0.09 \\
$\geq 2500 \mathrm{~g}$ & $72(82.76 \%)$ & $15(17.24 \%)$ & &
\end{tabular}

1.The brain MRI result's abnormal ratio of the mild group is $31.25 \%$, the moderate group is $16.33 \%$ and the severe group is $21.05 \%$, but the comparison of brain MRI results of the three neonates groups (Pख0.05) indicates that the difference is not statistically significant. 2.The brain MRI result's abnormal ratio of the pre-term is $30.77 \%$ and the full-term group is $16.88 \%$, but the comparison of brain MRI results between prem-term group and full-term group (Pख0.05) indicates that the difference is not statistically significant. 3.The brain MRI result's abnormal ratio of the $\triangle 2500 \mathrm{~g}$ group is $37.50 \%$ and the $\geq 2500 \mathrm{~g}$ group is $17.24 \%$; but the comparison of brain MRI results of two neonates groups (Pख0.05) indicates that the difference is not statistically significant.

2.2.4 Comparison of abnormal MRI signal values of globus pallidus on T1WI in mild, moderate and severe groups and comparison of these abnormal MRI signal values with normal MRI signal values Table 4 


\section{Group n $\quad$ Mean signal value of left globus pallidus}

Normal group ${ }^{1} \quad 82 \quad 892.03 \pm 132.54$

\begin{tabular}{|lccc|}
\hline Mild group ${ }^{2}$ & 5 & $956.85 \pm 245.87$ & $942.06 \pm 232.60$ \\
\hline $\begin{array}{l}\text { Moderate group } \\
23\end{array}$ & 8 & $1056.23 \pm 254.21$ & $1032.32 \pm 222.32$ \\
\hline
\end{tabular}

Mean signal value of right globus pallidus

$878.65 \pm 126.43$

$1239.03 \pm 288.53$

1. The comparison of normal group signal values with that of mild group $(p<0.05)$, with that of moderate group and with that of severe group $(p<0.01)$ indicates that the difference is statistically significant; 2 . The comparison of signal values between mild and moderate groups $(p<0.05)$ and between mild group and severe group $(p<0.01)$ indicates that the difference is statistically significant; 3 . The comparison of signal values between moderate group and severe group $(p<0.05)$ indicates that the difference is statistically significant.

2.2. 5 Comparison of BAEP testing results for neonates patients with abnormal MRI in three groups and comparison of hearing screening results between neonates patients with abnormal MRI and normal MRI.

Table 5

Group $\begin{array}{ll}\text { Normal } & \text { Abnormal MRI in } \\ \text { MRI } & \text { mild group[5] }\end{array}$

Abnormal MRI in moderate group $[8[$
Abnormal MRI in severe group $\square 8 \square$

प82П

\begin{tabular}{lllll}
\hline Normal & 67 & 3 & 4 & 2 \\
\hline Abnormal & 15 & 2 & 4 & 6 \\
\hline$\%$ & 18.29 & 40.00 & 50.00 & 75.00 \\
\hline Reviewabnormality & 0 & 0 & $1 \rrbracket 12.50 \rrbracket$ & $2 \varangle 25.00 \rrbracket$
\end{tabular}

\section{after one month}

1. There were 27 cases in abnormalitis of the BAEP results of all 103cases bilirubin patients. 2 . There were 15 cases in abnormalities of the BAEP result of the 82 cases normal brain MRL , 2 cases in abnormalities of the BAEP result of the 5 cases abnormal MRI in mild bilirubin group, 4 cases in abnormalities of the BAEP result of the 8 cases abnormal MRI in moderate bilirubin group and 6 cases in abnormalities of the BAEP result of the 8 cases abnormal brain MRI in severe bilirubin group. 3. After one month review of the BAEP result, there was 0 abnormal case in the normal MRI and the mild group; there were 1 abnormal case in the moderate group and 2 cass in the severe group. 
A. A Newborn with birth weight of $3.05 \mathrm{~kg}$, with normal delivery at $36+3$ weeks and clear amniotic fluid, Apgar score of 1 '-9', 5 '-9'; The serum total bilirubin was $502.3 \mathrm{~mol} / \mathrm{L}$ on the 6 th day after birth. Routine MRI of the basal ganglia examined on the 9th day after birth showed equal signal or slightly higher signal on T1WI, and the signal of globus pallidus was slightly higher than other nuclei in the basal ganglia on T1WI.

B. A Newborn with birth weight of $3.50 \mathrm{~kg}$, with normal delivery at $40+4$ weeks and amniotic fluid冈pollution, Apgar score of 1'-9', 5 '-9'; The serum total bilirubin was $130.4 \mathrm{~mol} / \mathrm{L}$ on the 8 th day after birth. Routine MRI of the basal ganglia examined on the 9th day after birth showed high signal on T1WI, and the signal of globus pallidus was symmetrical high signal on T1WI.

C. A Newborn with birth weight of $3.25 \mathrm{~kg}$, with normal delivery at $37+6$ weeks and clear amniotic fluid, Apgar score of 1 ' -8 ', 5 '-9'; The serum total bilirubin was $278.3 \mathrm{~mol} / \mathrm{L}$ on the 5 th day after birth. Routine MRI of the basal ganglia examined on the 8th day after birth showed high signal on $\mathrm{T} 1 \mathrm{WI}$, and the signal of globus pallidus was symmetrical high signal on T1WI.

D. A Newborn with birth weight of $2.87 \mathrm{~kg}$, with normal delivery at $38+5$ weeks and clear amniotic fluid, Apgar score of 1 ' -8 ', 5 '-8'; The serum total bilirubin was $478.6 \mathrm{~mol} / \mathrm{L}$ on the 7 th day after birth. Routine MRI of the basal ganglia on the 10th day after birth showed high signal on T1WI, and the signal of globus pallidus was symmetrical high signal on T1WI.

E. A Newborn with birth weight of $3.31 \mathrm{~kg}$, with normal delivery at 40 weeks and clear amniotic fluid, Apgar score of 1 '-8', 5 '-9';The serum total bilirubin was $327.3 \mathrm{~mol} / \mathrm{L}$ on the 7 th day after birth. Routine MRI of the basal ganglia at 10 postnatal days showed uneven signals in bilateral basal ganglia. Routine MRI of the basal ganglia revisited at 2 months showed high signals on $\mathrm{T} 2 \mathrm{WI}$, and the signal of globus pallidus was symmetrical high signals on T2WI.

\section{Discussion}

Neonatal jaundice is the most common clinical manifestation occurred in neonate period, which is mainly caused by the increase of serum bilirubin in neonates. In the prenatal period, due to the hypoxic environment, the amount of red cells is increased and the hemoglobin content in the fetal is high . After birth, due to the hyperoxic environment, the survival time of red blood cell becomes short. The fetal hemologlobin is gradually converted to adult hemoglobin producing a large amount of bilirubin, which exceeds the ability of liver to treat bilirubin. When bilirubin levels are above $139 \mu \mathrm{mol} / \mathrm{L}$, skin or organ 
xanthochromia may appear, that is called neonate jaundice. It has been previously thought if the concentration of bilirubin in the blood is above $342 \mu \mathrm{mol} / \mathrm{L}$, serious damage to nervous system for the neonates may occur and the acute stage manifestation of acute bilirubin encephalopathy (ABE) or chronic kernicterus and BIND appears ${ }^{[9,10]}$. Brain damage caused by neonatal hyperbilirubinemia(NHB) is not always reversible, may lead to cerebral palsy and hearing loss. The most common type of nerve injuries are the sequelae of severe neurological dyskinesia and hearing impairment ${ }^{[2,11-13]}$. Acute bilirubin encephalopathy or kernicterus and BIND mainly affects subcortical regions, such as globus pallidus, hypothalamus, substantia nigra, cerebellar dentate nucleus, or hippocampus, brainstem, etc ${ }^{[14,15]}$. Although, the underlying mechanisms are still unknown ${ }^{[16,17]}$. Most of ABE neonatal patients still have a chance to be recovered by timely treatment of reducing serum bilirubin levels, while only a few suffer from sequelae, like kernicterus.

The feature of MRI manifestation to bilirubin encephalopathy is the high signal of globus pallidus in the acute stage of T1-weighted imaging (T1WI). With the development of the disease, it has changed from the high signal in acute stage of T1WI to the high symmetric signal of bilateral globus pallidus and subthalamic nucleus in chronic stage and FLAIR sequence signal ${ }^{[18-20]}$. Since in the neonatal period the basal ganglia nerve cells have strong physiological and biochemical metabolism and the oxygen consumption is increasing, especially, the middle and later part of the globus pallidus is the most sensitive ${ }^{[21]}$. This leads to the selectively deposition of serum bilirubin in the globus pallidus, the middle and later part of the globus pallidus is more sensitive ${ }^{[21-23]}$. Besides, it will cause the damage to neurons and glial cells, the apoptosis of neurons and the change of glial cells' mitochondria function ${ }^{[24,25]}$. Basal ganglia pallidus injury caused by hyperbilirubinemia can be efficiently detected by MRI. The high and symmetric signal of bilateral T1WI pallidal is an important imaging feature of neonatal $A B E$, while the change from high signal of T1WI to high signal of T2-weighted imaging (T2W1) is the imaging feature of nuclear jaundice on MRI, indicating neuronal cell necrosis and poor prognosis ${ }^{[26]}$. Presently, the mechanism of MRI signal change still remains unclear. This may be related to the reduction of the T1 value, which is caused by the deposition of bilirubin in glial cells and the destruction of bilirubin to nerve cell membrane ${ }^{[27]}$.

Most of the MRI results mentioned above were obtained from brain MRI findings of the severe or profound NHB patients with bilirubin encephalopathy or non-bilirubin encephalopathy. So far, there is no report about whether the MRI results of mild and moderate NHB patients without bilirubin encephalopathy manifestation are abnormal. Therefore, this study retrospectively analyzes the bilirubin level and MRI results of 103 patients with jaundice in order to understand whether there is abnormal brain MRI at different bilirubin levels, as well as whether there is statistical difference of abnormal brain MRI results in bilirubin levels, gestational age, and birth weight.

It is reported in literature that some mild levels of bilirubin could cause temporary or permanent neurological sequelae under the condition that a certain level of bilirubin is considered as being safe by people ${ }^{[28]}$. Based on total serum bilirubin concentration(TSB), the 103 patients in our research were 
divided into three groups, including 16 cases in the mild group (TSB: $0.0-229.0 \mu \mathrm{mol} / \mathrm{L}$ ), 49 cases in the moderate group (TSB: $229.0-342.0 \mu \mathrm{mol} / \mathrm{L}$ ) and 38 cases in the severe group ( TSB $\geq 342.0 \mu \mathrm{mol} / \mathrm{L}$ ). There were 21 cases with abnormal MRI results, which consisted of 5(31.25\%)cases in mild group, $8(16.33 \%)$ cases in moderate group and $8(21.05 \%)$ cases in severe group(Table 3$)$. The comparison of TSB among the three groups indicated that the difference was statistically significant (P凶0.01)(Table 1), whereas, the comparison of abnormal brain MRI results among the three groups indicated that the difference was not statistically significant $(P \otimes 0.05)$. This pointed out that bilirubin brain nerve damage may occur in patients without the manifestation of ABE even in low level of bilirubin, and the rate of MRI abnormality is not rising with the rising of bilirubin level. The study findings are not consistent with the reports of El Houchi, S.Z. et al. that the higher the total bilirubin level is, the higher the proportion of abnormal MRI is ${ }^{[29]}$. We think that low number of cases in the mild group may also be an important factor. Under Taoka 140 et al.'s follow-up of the observed subjects for 2 years, it was found that the infants had been developing normally whether the high symmetric T1WI signal was demonstrated in globus pallidus (GP) and substantia niga (STN) regions or not. In accordance with reports in the literature, it may be related to the development of gray matter mass in GP and STN after the birth of neonates ${ }^{[30-33]}$. Besides, lots of literatures have reported that this manifestation is one of the brain MRI findings of nuclear jaundice ${ }^{[34-36]}$. Along with the extensive clinical application of MRI, the manifestation is becoming more and more common in neonates, and normal neonates. Thus, some scholars recently have raised different opinions ${ }^{[29-31]}$. For example, Harris et al. ${ }^{[37]}$ reported that high symmetric signal in the globus pallidus region of four 5-21 day neonates with acute kernicterus has disappeared during the follow-up. This manifestation was thought transient, had no correlation with the prognosis of the patients. This manifestation also appeared in the cases of neonatal hypoxic ischemic encephalopathy, hypoglycemia and other cases ${ }^{[38,39]}$.

In this study, the neonate patients, with high signal of globus pallidus in T1WI or T2W1, caused by the diseases of neonatal hypoxic ischemic encephalopathy, hypoglicemia, hepatolenticular degeneration and other diseases, have been excluded. Of our 21 patients with MRI, 12 were followed up for one month, and 9 cases were followed up for 3 months. 6 cases of patients had normal brain MRI results in the 1 month later reexamination. 3 cases of patients had not been reexamined of the MRI, one of the 3 cases had neurological abnormalities 2 months later, who was delivered with gestational age 40 weeks, birth weight $3.31 \mathrm{~kg}$, clear amniotic fluid, the Apgar score 8 at 1 min and 9 in 5 mins, maximum bilirubin Value $327.3 \mu \mathrm{mol} / \mathrm{L}$, and uneven signal of the brain MRI in bilateral basal ganglia region. However, one case of this study showed no brain MRI abnormalities with maximum bilirubin value $502.3 \mu \mathrm{mol} / \mathrm{L}$, birth weight $3.05 \mathrm{~kg}$, gestational age 36+3 weeks, clear amniotic fluid and the Apgar score 9 at $1 \mathrm{~min}, 9$ in $5 \mathrm{mins}$. In addition, another case in the study may have ABE with gestational age $40+4$ weeks, birth weight $3.5 \mathrm{~kg}$, meconium stained amniotic fluid during birth, the Apgar score 9 at $1 \mathrm{~min}, 9$ in 5 mins, the maximum bilirubin value $130.4 \mu \mathrm{mol} / \mathrm{L}$, and high symmetric T1WI signal of bilateral basal ganglia indicated by his brain MRI. The high symmetric signal of bilateral globus pallidus is not unique to the neonatal $A B E$, it can also be seen in some neonatal patients with hypoxic ischemic encephalopathy ${ }^{[40,41]}$, or even in normal neonates. But the MRI manifestations of hypoxic ischemic encephalopathy(HIE) involved more extensive 
scope, which was characterized by internal capsule, the putamen and the thalami, and accompanied by cortical and subcortical, deep leukoplakia plaque abnormal signals, diffuse brain edema, intracranial hemorrhage, and so on. These accompanied manifestations were rarely reported in bilirubin encephalopathy ${ }^{[42]}$.

Blood brain barrier injury, might be caused by anoxia or other factors in antepartum and intrapartum, increased the permeability of blood brain barrier, resulting in the increasing of free bilirubin to enter into brain tissue through the injured blood-brain barrier and to deposit in the basal nerve nucleus, cerebral ganglia, subthalamic nucleus, parietal nucleus, ventricular nucleus, caudate nucleus, cerebellum, oblongata, cerebral cortex , spinal cord and brainstem, etc ${ }^{[43]}$. Accordingly, the utilization of oxygen in brain tissue was inhibited, 176 leading to brain damage. It is also believed that bilirubin deposition cause the influx of neuron cells $\mathrm{Ca} 2{ }^{[44]}$, stimulate the increase of proteolytic enzyme activity ${ }^{[45]}$, oxidative damage ${ }^{[46]}$, immune stimulation, immunotoxicity ${ }^{[47]}$, increased cytoglutamate and excitotoxicity ${ }^{[48]}$, imflammatory damage ${ }^{[49]}$ and other pathways, leading to neuronal necrosis and apoptosis ${ }^{[41]}$. It has been reported in many studies that the occurrence of neonatal BE is related to the factors of gestational age $^{[50]}$, birth weight ${ }^{[51]}$, bilirubin binding state and bilirubin level ${ }^{[52]}$, etc. In this study, 103 patients were divided into preterm group ( 26 cases) and full-term group ( 77 cases), observing the comparison of bilirubin values $(289.70 \pm 85.38 \mathrm{vs} 310.36 \pm 72.32, \mathrm{P}=0.232)$ of the two groups and the MRI abnormal result $(P=0.16)$ of the two groups was $P \otimes 0.05$, the difference was not statistically significant, which indicated that there may be no difference in the toxicity of bilirubin to central nervous system between pre-term group and full-term group without BE clinical manifestation. Moreover, it did not appear to be the premature infants with younger gestational age were more susceptible to bilirubin toxicity. The analyses of this result may be as follows: (1) Premature delivery patients, who are usually admitted to be in hospital after birth, are treated for jaundice in time during hospitalization. While, full-term infants are admitted to be in hospital only when their bilirubin value reaches a higher level. On the one hand, high level of bilirubin could easily cause nerve damage by through the blood brain barrier; on the other hand, the longer the high level of bilirubin remains in the body, the more neurotoxicity of bilirubin is ${ }^{[53]}$. (2) The statistical analysis in this study may be biased, on account of the fewer cases of pre-term delivery group, especially lack of pre-term patients less than 31 weeks of gestational age due to obstetric factors. Meanwhile, we also observed whether there was statistical difference between birth weight and brain MRI abnormality of the jaundice patients. Based on birth weight, 103 patients were divided into two groups, one group birth weight $<2500 \mathrm{~g}$ ( 16 cases, including 6 abnormal cases) and the other group birth weight $\geq 2500 \mathrm{~g}$ (87 cases, including 15 abnormal cases) (Table 2 ). The comparison of brain MRI abnormality of the two groups was $P=0.09, P>0.05$, the difference was not statistically significant.

In addition, the comparison of bilirubin value between abnormal MRI group and normal MRI group was(303.56 \pm 83.04 vs $305.55 \pm 74.54, P=0.92, P>0.05$ )(Table 1 ), the difference was not statistically significant, which indicated that there was no obvious difference in bilirubin level between abnormal MRI group and normal MRI group of jaundice patients without ABE. 
At the same time, we measured the T1WI signal values of the patients with abnormal brain MRI and the signal values of the patients with normal brain MRI in the three groups, and performed statistical comparison, which showed that the T1WI signal values of the patients with abnormal MRI were all higher than those of the patients with normal MRI $(P<0.05)$, and the T1WI signal values of the patients with MRI abnormalities in the three groups also had statistically difference $(P<0.05)($ Table 4$)$. With the increase of serum bilirubin level, the T1WI signal value of the patients with MRI abnormalities also increased, which was consistent with the report of Yan Ruifang et al ${ }^{[54]}$ that there was a linear correlation between the mean signal value of globus pallidus and serum total bilirubin levels in the lesion group. This indicated that with the rising of bilirubin level, the more bilirubin deposited on neuron such as globus pallidus, the more serious damage would be made to nerve tissue such as neuron.

NHB could not only lead to acute bilirubin encephalopathy and kernicterus but also bilirubin induced neurological dysfunction (BIND) ${ }^{[55,56]}$, which includes mild neurological abnormalities, cognitive disorder, auditory neuropathy spectrum disorder(ANSD) ${ }^{[57]}$ and so on. Auditory complications, a disabling neurological finding in kernicterus, are typically characterized by varying degree of auditory neuropathy/dys-synchrony (AN/AD) ranging from central auditory processing difficulties with normal hearing to severe AN/AD with absent auditory brainstem responses, and possibly accompanying severe hearing loss and deafness. In fact, the brainstem cochlear nuclei are said to be one of the first structures affected by elevated total bilirubin, followed by the auditory nerve ${ }^{[58,59]}$. So we performed hearing tests for the 103 patients with brainstem auditory evoked potential (BAEP) ${ }^{[60]}$ devices. From table 5 , it was found that 15 cases of 82 patients with normal MRI results were abnormal, accounting for $18.29 \%$. In the mild group 2 cases of 5 patients with abnormal MRI were abnormal, accounting for $40.00 \%$. In the moderate group 4 cases of 8 patients with abnormal MRI were abnormal, accounting for $50.00 \%$. In the severe group 6 cases of 8 patients with abnormal MRI were abnormal, accounting for $75.00 \%$. The change of BAEP is closely related to bilirubin concentration and duration, but BAEP may be abnormal in children with moderate bilirubin level, that is, BAEP may be abnormal in children with related safe bilirubin ormalities were improved after clinical treatment. concentration even if there is no clinical manifestion ${ }^{[61,62]}$. BAEP was improved with the decrease of serum bilirubin or after the process intervention such as phototherapy and blood exchange transfusion ${ }^{[63-65]}$. Vitoria Akinpelu, et al ${ }^{[61]}$. reported in 2013 that the abnormal BAEP rate fluctuated between $9 \%$ and $83.3 \%$ before clinical intervention, and about over half of the BAEP abnormalities were improved after clinical treatment. After admission, all 103 patients were treated with medication and/or phototherapy, and their jaundice decreased significantly. After 1-3 month followed-up, the reexamination found that the patients with hearing abnormalities in the MRI normal group and the mild group were all returned to normal level, and 1 case in the moderate group and 2 cases in the severe group were remained abnormal. Sharma ${ }^{[66]}$, et al. and Agrawa [67], et al. showed that BAEP improved in $77.2 \%$ and $76.5 \%$ children. This result indicated that, on the one hand, the BAEP abnormal rate of the patients with abnormal MRI was higher than that of the patients with normal MRI, and the BAEP abnormal rate of the patients with abnormal MRI increased with the increase of bilirubin level. It also shows that with the increase of bilirubin, the hearing damage is more obvious ${ }^{[68]}$. On the other hand, it also proved the application value of MRI in neonatal jaundice patients, which could timely detect the 
abnormalities of central nervous system of the patients with jaundice, and provide imaging evidence for early diagnosis and early intervention.

Based on this study, we found that there were abnormalities in the neonatal brain's MRI and BAEP in the different bilirubin concentration levels. It indicates that in some conditions, even at the low level of bilirubin concentration might lead to neurological damage in the neonatal period. So we strengthen the monitoring of neonatal jaundice and may use the MRI and BAEP to test the bilirubin induced neurological damage for neonataes.

Limitations of the Present Study

Although the findings of this study indicate that in babies with jaundice, the brain MRI may show abnormal changes at mild and moderate levels of bilirubin, and there is no statistically significant difference between brain MRI abnormalities in the severe group, Our results have been affected by a number of issues. First, we failed to measure the free bilirubin levels, internal environmental conditions such as serum albumin levels, $\mathrm{pH}$ values, etc. of the jaundice newborns participating in the study at that time, so it cannot be said with certainty that the baby's brain was exposed to bilirubin crossing the bloodbrain barrier Prime type. Secondly, the sample size in this study is relatively small, especially in the mild group and the group less than $2500 \mathrm{~g}$. Finally, the craniocerebral pathology test of newborn animal Jaundice model and long-term follow-up is lacking to validate our findings. This will be an issue that we need to address in this type of research in the future.

In conclusion, in the presence of certain factors, such as premature, hypoproteinemia, potential intrauterine hypoxia, etc, central nervous system damage may also occur at low level of bilirubin and result in abnormality on MRI and BAEP. Meanwhile, MRI and BAEP can also be used to provide early abnormal information for the judgment of central nervous system damage to NHB neonatal patients without clinical manifestations of $A B E$, and offer clues for early treatment and early intervention so as to prevent the occurrence of severe brain tissue damage .

\section{Abbreviations}

$A B E$ : acute bilirubin encephalopathy

TSB : total serum bilirubin

MRI: Magnetic resonance imaging

NHB : neonatal hyperbilirubinemia

$A B E$ : acute bilirubin encephalopathy

T1WI :T1-weighted imaging

T2WI :T2-weighted imaging 


\section{Declarations}

Declarations: We have no specific state here.

Ethics approval and consent to participate: The study was approved by the Ethics Committee of the First People's Hospital of Taicang City. All parents of participants provided written informed consent for participation in this study.

Consent to publish: Our authors agree to publish this article.

Competing interests: The authors claim no conflict of interest.

Funding : There is no funding source.

Authors' Contributions:

Z: Responsible for project writing/execution and paper writing.

S and F: Participate in project execution and data collection.

$\mathrm{H}$ : Give guidance on this subject and revise the paper.

Acknowledgements: We would like to express our heartfelt thanks to all our colleagues for their assistance in this subject.

\section{References}

1. Olusanya, B.O.; Ogunlesi, T. A.; Slusher, T. M. Why is kernicterus still a major cause of death and disability in low-income and middle-income countries[J]? Arch. Dis. Child. 2014,99,1117-1121.

2.Shaprio, S.M. Chronic bilirubin encephalopathy: Diagnosis and outcome[J]. Semin. Fetal Neonatal Med. 2010,15, 157-163.

3. Uziel, A.; Marot, M.; Pujol, R. The Gunn rat: An experimental model for central deafness[J]. Acta Otolaryngol.1983, 95, 651-656.

4. Olds, C.; Oghalai, J.S. Bilirubin-Induced Audiologic Injury in Preterm Infants[J]. Clin. Perinatol. 2016, 43,313-323.

5. Wiskownski, J.L.; Panigraphy, A.; Painter, M.J.; et al.Magnetic resonance imaging of bilirubin encephalopathy: Current limitations and future promise[J]. Semin. Perinatol. 2014, 38, 422-428.

6.Okumura A. Hayakawa F. Maruyama K, et al. Single photo emission computed tomography and serial MRI in preterm infants with kernicterus[J]. Brain Dev, 2006,28(6): 348-352. 
7. N Y Boo M. Oakes M S. Lye H. Said, Risk factors associated with hearing loss in term neonates with hyperbilirubinaemia[J], J. Trop. Pediatr, 1994, 40:194-197.

8.Muchowski, K.E. Evaluation and treatment of neonatal hyperbilirubinemia[J]. Am. Fam. Physician 2014, $89,873-878$.

9. Olusanya, B.O.; Ogunlesi, T.A.; Slusher, T.M. Why is kernicterus still a major cause of death and disability in low-income and middle-income countries[J]? Arch. Dis. Child. 2014, 99, 1117-1121.

10. Olusanya, B.O.; Kaplan, M.; Hansen, W.R. Neonatal hyperbilirubinemia: A global perspective[J]. Lancet Child Adolesc. Health 2018, 2, 610-620.

11. Shapiro SM. Kernicterus. In: Stevenson DK, Maisels MJ,Watchko JF, editors. Care of the Jaundiced Neonate [J].New York: McGraw-Hill; 2012. p. 229-242.

12. Shapiro SM, Popelka GR. Auditory Impairment in Infants at Risk for Bilirubin-induced Neurologic Dysfunction[J]. Semin Perinatol. 2011; 35: 162-170.

13. Campistol J, Galvez H, Cazorla AG, et al. Neurological Dysfunction Induced by Bilirrubin[J]. Neurologia, 2012; 27: 202-211.

14. López-Corella, E.; Ibarra-González, I.; Fernández-Lainez, C.; et al. Kernicterus in a boy with ornithine transcarbamylase defificiency: A case report[J]. Neuropathology 2017, 37, 586-590.

15. Adle-Biassette, H.; Harding, B.; Golden, J.A. Developmental Neuropathology; John Wiley and Sons Ltd.: Oxford, UK, 2018.

16. Watchko JF, Painter MJ, Panigrahy A. Are the Neuromotor Disabilities of Bilirubin-induced Neurologic Dysfunction Disorders Related to the Cerebellum and Its Connections? [J] Semin Fetal Neonatal Med 2015, 20: 47-51.

17. Wisnowski JL, Panigrahy A, Painter MJ, et al. Magnetic Resonance Imaging of Bilirubin Encephalopathy: Current Limitations and Future Promise[J]. Semin Perinatol 2014;38:422-428.

18. Parashari UC, Singh R, Yadav R, et al. Changes in the Globus Pallidus in Chronic Kernicterus [J]. J Pediatr Neurosci 2009;4: 117-119

19. Magalha es MOGM, Brası 'lia DF. Kernicterus, Chronic Bilirubin Encephalophy: Imaging Features and Clinical Correlation[J]. ECR2016/ C-2261.

20. Ribeiro BN, Lima GA, Ventura N, et al. Chronic Kernicterus: Magnetic Resonance Imaging Findings[J]. Radio Bras 2016;49:407e8.

21. Ribeiro BN, Lima GA, Ventura N, Gasparetto EL, Marchiori E. Chronic Kernicterus: Magnetic Resonance Imaging Findings[J]. Radio Bras 2016;49:407e8. 
22. Cece H, Abuhandan M, Cakmak A,et al. Diffusion-weighted Imaging of Patients with Neonatal Bilirubin Encephalopathy[J]. Jpn J Radiol 2013;31:179-185.

23. Gkoltsiou K, Tzoufi M, Counsell S, et al. Serial Brain MRI and Ultrasound Findings: Relation to Gestational Age, Bilirubin Level, Neonatal Neurologic Status and Neurodevel-opmental Outcome in Infants at Risk of Kernicterus[J].Early Hum Dev 2008;84:829-838

24. Brito,M.A.,Silva,R.F.,andBrites, D.Bilirubin induces loss of membrane lipids and exposure of phosphatidylserine in humanery- throcytes[J]. CellBiol.Toxicol. 2002,18, 181-192.

25. Falcão,A.S.,Silva,R.F.,Pancadas, S., et al. Apoptosisand impairment of neuritenet work by short exposure of immature ratcor- tical neuron stoun conjugated bilirubin increase with cell differentiation and are additionally enhanced by an inflammatory stimulus[J]. J. Neurosci. Res.2007, 85, 1229-1239.

26. Okumura Akihisa, Kidokoro Hiroyuki, Shoji Hiromichi, et al. Kernicterus in preterm infants[J]. Pediatrics, 2009, 123 (6): e1052-1058.

27. Amit Y, Poznansky MJ, Schiff D: Neonatal jaundice and bilirubin encephalopathy: a clinical and experimental reappraisa[J]I. Isr J Med Sci 1992, 28:103-108.

28. Govaert P, Lequin M, Swarte R, et al. Changes in Globus Pallidus with (pre)term Kemicterns [J].Pediatrics, 2003, 112(6 Pt 1)I1256 -1263

29. El Houchi, S.Z.; Iskander, I.; Gamaleldin, R.; et al. Prediction of 3- to 5-Month Outcomes from Signs of Acute Bilirubin Toxicity in Newborn Infants[J]. J. Pediatr. 2017, 183, 51-55.

30. Taoka T, Aida N, Ochi T, et al. Transient Hyperinteusity in the Subthalamic Nucleus and Glohus Pallidus of Newborns on T1-weighted images [J]. AJNR Am J Neuroradiol, 2011, 32(6):1130-1137囚

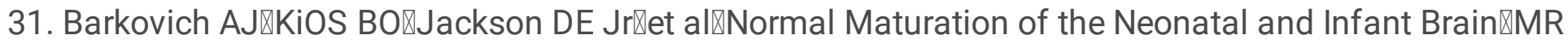
Imaging at 1.5T [J]. Radiology, 1988, 166(1 Pt 1):173-180.

32. Counsell SJ,Maalouf EF, Fletcher AM, et al. MR Imaging Assessment of Myelination in the Very Preterm Brain [J]. AJNR Am J Neuroradiol, 2002,23(5):872-881.

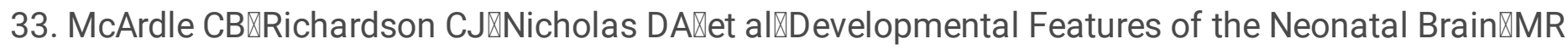
Imaging. Part IهGray-white Matter Differentiation and Myelination [J]. Radiology, 1987, 162 (1 Pt 1):2232298

34. Penn AA, Enzmann DR, Hahn IS, et al. Kemictems in a Full-term Infant [J]. Pediatrics, 1994, 93(6 Pt 1):1003-1006ه

35. Govaert P, Lequin M, Swarte R, et al. Changes in Globus Pallidus with (pre)term Kemicterns [J].Pediatrics, 2003, 112(6 Pt 1)|1256 -1263】 
36. Coskun A, Yikilmaz A, Kumand $\infty$ S, et al. Hyperintense Globus Pallidus on T1-weighted MR Imaging in Acute Kernicterus: Is It Common or Rare? [J]. Eur Radial, 2005, 15(6):1263-1276区

37. Harris MC囚Bembaum JC『Polin JR囚et al. Developmental Followup of Breastfed term and Near-term Infants with Marked Hyperbilirubinemia [J]. Pediatrics, 2001, 107(5):1075-1080

38. De Vries LS, Groenendaal F. Patterns of Neonatal Hypoxic Ischaemic Brain Injury [J]. Neumradiology, 2010, 52(6):555-566区

39. Bekiesinska. Figatowska M, Mierzewska H, et al. Basal Ganglia Lesions in Children and Adults [J]. Eur J Radiol, 2013, 82(5):837-849

40. Cece $\mathrm{H}$, Abuhandan $\mathrm{M}$, Cakmak A, et al. Diffusion-Weighted Imaging of Patients with Neonatal Bilinlbin Encephalopathy [J].Jpn J Radiol, 2013, 31(3):179-185

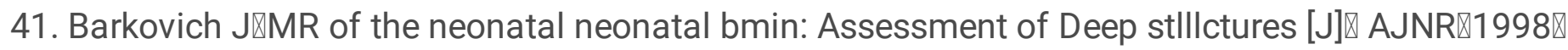
19(8): 1397-1403.

42. K. Kaga, M. Suzuki, S. Koyama, Neonatal asphyxia and hyperbilirubinemia[J].JOHNS 16 (2000) 1695 -1699 .

43. .Nakamura $\mathrm{H}$, Takada $\mathrm{S}$, Shimabuku $\mathrm{R}$, et al. Auditory nerve and brainstem responses in newborn infants with hyperbilirubinemia[J]. Pediatrics, 1985, 75:703-708.

44.Zhang, B., Yang, X., and Gao, X. . Taurine protects against bilirubin induced neurotoxicity in vitro[J]. Brain Res. 2010,1320, 159-167.

45.Vaz, A. R., Silva, S. L., Barateiro, A., et al. Proinflflammatory cytokines intensify the activation of NO/NOS, JNK1/2 and caspase cascades in immature neurons exposed to ele-vated levels of unconjugated bilirubin[J]. Exp. Neurol.2011, 229, 381-390.

46.Hansen, T. W., Allen, J. W., and Tom marello, S. (1999). Oxidation of bilirubin in the brain-further characterization of a potentially protective mechanism[J]. Mol. Genet. Metab. 68, 404-40

47.Falcão, A. S., Fernandes, A., Brito, M. A., et al. Bilirubin-induced immunostimulant effects and toxicity vary with neural cell type and maturation state[J]. Acta Neuropathol. 2006,112, 95-105.

48. Silva, S. L., Vaz, A. R., Diógenes, M. J., et al. Impairment of neurite sprouting by unconjugated bilirubin is mediated by $\mathrm{NO}$ and glutamate,modulated by microglia, and prevent-ed by glycoursodeoxycholic acid and interleukin-10[J]. Neuropharmacology, 2012, 62, 2397-2407.

49.Fernandes, A., Falcão, A. S., Silva, R. F.,et al. Inffflammatory signalling pathways involved in astroglial activation by unconjugated bilirubin[J]. J. Neurochem.2006, 96, 1667-1679. 
50.Muchowski KE. Evaluation and Treatment of Neonatal Hyperbilirubinemia[J]. Am Fam Physician. 2014 ;89(11):873-878.

51.Vidit Bhargava, Daniel Tawfik, Bruce Niebuhr, et al. Transcutaneous Bilirubin Estimation in Extremely Low Birth Weight Infants Receiving Phototherapy: A Prospective Ob-servational Study[J]. BMC Pediatr ,2018; 18 (1) :227.doi: 10.1186/s12887-018-1207-7.

52.El Houchi, S.Z.; Iskander, I.; Gamaleldin, R.;et al. Prediction of 3- to 5-Month Outcomes from Signs of Acute Bilirubin Toxicity in Newborn Infants[J]. J. Pediatr. 2017, 183, 51-55.

53.Gkoltsiou,K.,Tzoufi,M.,Counsell, S.,et al. Serial brain MRI and ultrasound findings:relation to gestational age, bilirubinlevel, neonatal neurologic status and neurodevelopmental outcome in infants at risk of kernicterus[J]. EarlyHum .Dev. 2008, 84, 829-838.;

54.Zhou Liu, Bing Ji,Yuzhong Zhang,et al.Machine Learning Assisted MRI Characterization for Diagnosis of Neonatal Acute Bilirubin Encephalopathy[J].Front Neurol. 2019; 10: 1018. doi:

10.3389/fneur.2019.01018

55.Shapiro SM. Definition of the clinical spectrum of kernicterus and bilirubin induced neurological dysfunction (BIND)[J]. J Perinatol,2005, 25 (1): 54-59.

56.Smitherman $\mathrm{H}$, Stark AR, Bhutan VK.Early recognition of neonatal hyperbilirubinemia and its emergent management[J]. Semin Fetal Neonatal Med, 2006, 11(3): 214-224.

57.Rapin I, Gravel J. "Auditory neuropathy" $₫$ physiologic and pathologic evidence calls for more diagnostic specificity[J]. Int J Pediatr Otorhinolaryngol, 2003,67(7): 230-246.

58.Uziel, A.; Marot, M.; Pujol, R. The Gunn rat: An experimental model for central deafness[J]. Acta Otolaryngol.1983, 95, 651-656.

59.Olds, C. Oghalai, J.S. Bilirubin-Induced Audiologic Injury in Preterm Infants[J]. Clin. Perinatol. 2016, 43,313-323.

60.Kimitaka Kaga , Eiji Kitazumi , Kazuo Kodama. Sensorineural hearing loss in patients with cerebral palsy after asphyxia and hyperbilirubinemia [J]. International Journal of Pe-diatric Otorhinolaryngology (2005) 69, 1211-1217

61.Olubunmi Victoria Akinpelu, Sofia Waissbluth, Sam J. Daniel. Auditory risk of hyperbilirubinemia in term newborns: A systematic review international[J]I Journal of Pediatric Otorlaryngology, 2013,77:898905.

62.K. Sheykholeslami K. Kaga. Otoacoustic emission and auditory response after neonatal hyperbilirubinemia[J], Int. J. Pediatr. Otorhinolaryngol, 2000,52:65-73. 
63.Perlman $\mathrm{M}$, Fainmesser $\mathrm{P}$, Sohmer $\mathrm{H}$, et al. Auditory nerve-brainstem evoked response in hyperbilirubinemic neonates[J]. Pediatrics, 1983, 72:658-664.

64.Nwaesei CG, Van Aerde J, Boyden M, et al. Changes in auditory brainstem responses in hyperbilirubinemic infants before and after exchange transfusion[J]. Pediatrics, 1984, 74(5): 800-803.

65.Wennberg RP, Ahlfors $\mathrm{CE}$, Bickers R, et al. Abnormal auditory brainstem response in a newborn infant with hyperbilirubinemia: improvement with exchange transfusion[J]. J Pediatr, 1982, 100 (4): 624-626.

66.V. K. Agrawal, R. Shukla, P. K. Misra, R. K. Kapoor, G. K. Malik, Brainstem auditory evoked response in newborns with hyperbilirubinemia[J], Indian Pediatr, 1998, 35: 513-518.

67.P. Sharma NP. Chhangani KR. Meena R, et al. Gupta, Brainstem evoked response audiometry (BAER) inneonates with hyperbilirubinemia[J], Indian J. Pediatr, 2006, 73: 413-416.

68. Funato M, Tamai H, Shimada S, et al. Vigitiphobia, unbound bilirubin, and auditory brainstem responses[J]. Pediatrics, 1994,93(1): 50-53.

\section{Figures}



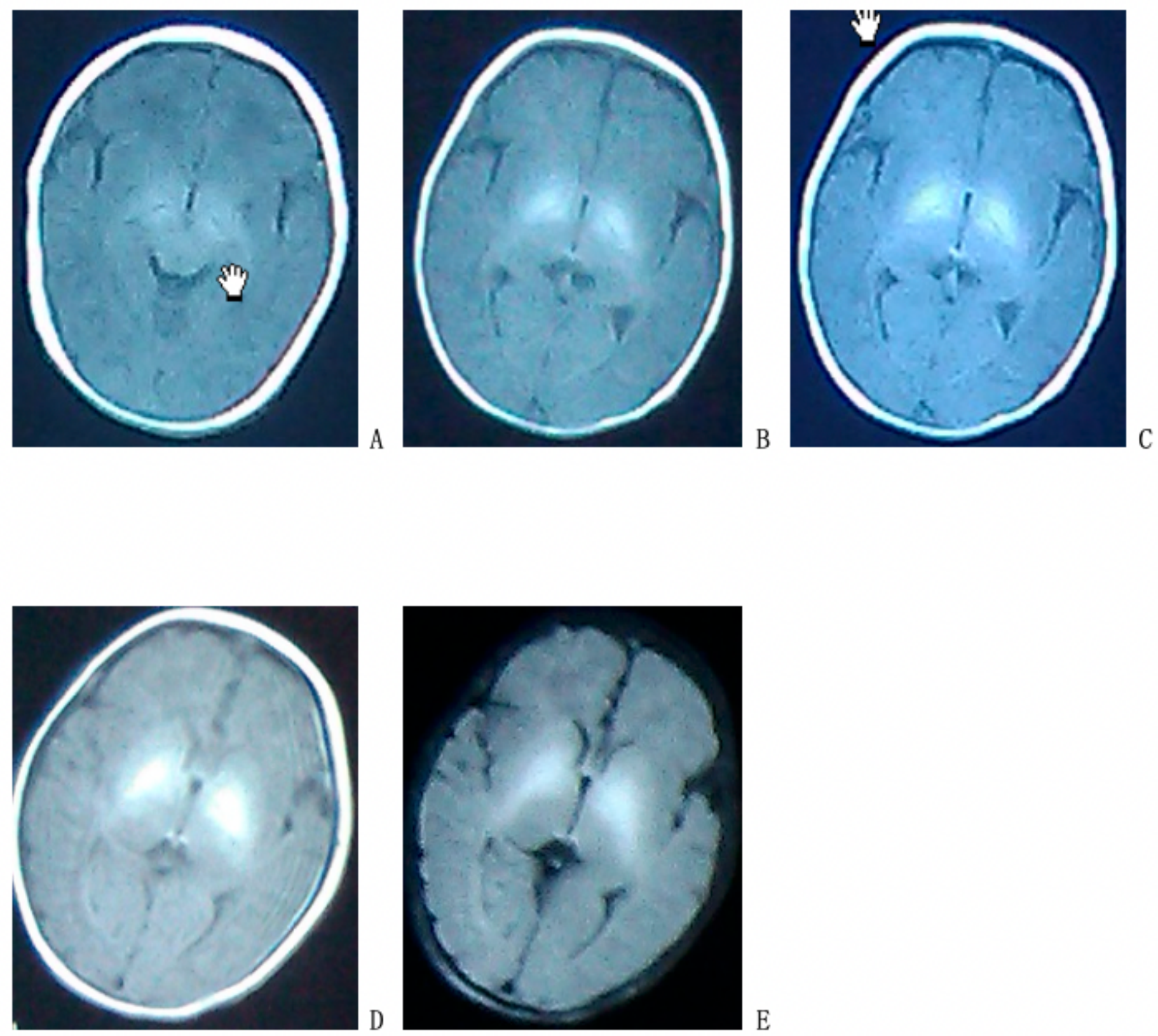

Figure 1

MRI pictures. A normal B mild C moderate D severe E T2WI manifestation 